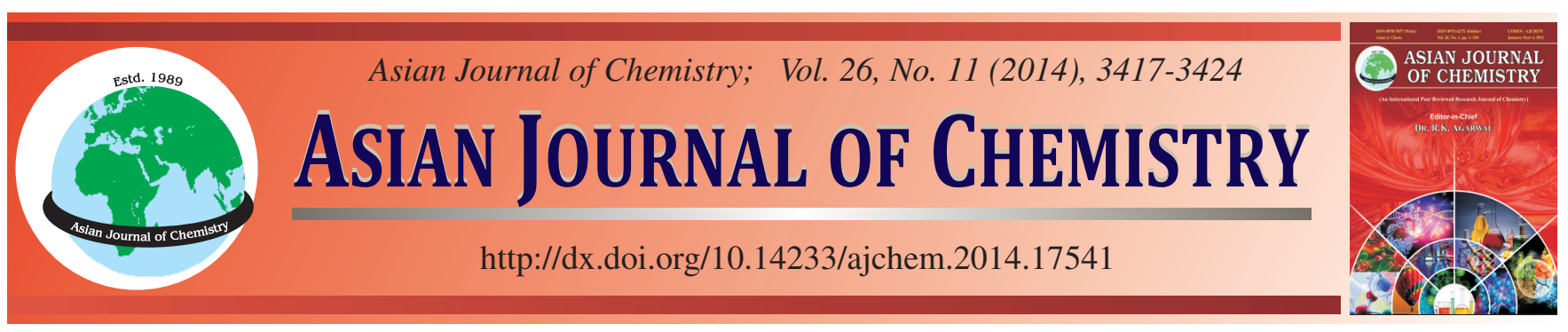

\title{
Generation of Insulin-Producing Cells from Bone Marrow Mesenchymal Stem Cells with Modified Expression of PDX1 and NKX6.1
}

\author{
Xiao-Long Tang ${ }^{1,2}$, Ze-Ming Wan ${ }^{3}$, Yun-Long Gao ${ }^{3}$, Cheng Zhang ${ }^{3}$, Xian-Zhang Huang ${ }^{3}$, \\ Li-FA Xu ${ }^{1}$, Zhen Huo ${ }^{1}$, Tian-Y I Chen ${ }^{1}$, Shu-Yu Cai ${ }^{1,2}$, Zhen-You Jiang ${ }^{4}$, Jia Chen $^{4}$, \\ Liu-Hua WeI ${ }^{5}$, JiAn Wang ${ }^{1, *}$ and XIAO-YIN $\mathrm{CheN}^{4}$
}

\begin{abstract}
${ }^{1}$ Stem Cell Engineering Research Center, School of Medical, Anhui University of Science \& Technology, Huainan 232001, P.R. China ${ }^{2}$ Nankai Hospital, Nankai Clinical School, Tianjin Medical University, Tianjin 300100, P.R. China

${ }^{3}$ Clinical Laboratory, Second Affiliated Hospital, Guangzhou University of Traditional Chinese Medicine (Guangdong Provincial Hospital of TCM), Guangzhou 510120, P.R. China

${ }^{4}$ Department of Microbiology and Immunology, School of Medical, Jinan University, Guangzhou 510632, P.R. China

${ }^{5}$ Clinical Laboratory, Liuzhou worker's Hospital, Guangxi 545005, P.R. China
\end{abstract}

*Corresponding author: Tel: +86 20 85226197; E-mail: txljd2006@126.com

\begin{abstract}
This study was designed to efficiently induce the differentiation of the bone marrow mesenchymal stem cells (BMSCs) into insulinproducing islet cells by modifying the expression of pancreatic and duodenal homeobox factor 1 (PDX1) and NKX6 transcription factor related 1 (NKX6.1). Bone marrow mesenchymal stem cells were infected by the recombinant adeno viruses carrying Pdx 1 and Nkx6.1 genes for differentiation and the differentiated cells were transplanted into the subrenal capsule of diabetic mice, then the secretion of insulin and C-peptide were examined. The effects of the differentiated cells were also investigated through measuring the blood glucose levels. The BMSCs induced by recombinant adeno virus of pAdxsi-CMV-PDX1/CMV-NKX6.1 and several cytokines showed positive dithizone staining and significant expression of insulin and glucose transporter-2. Stimulation with different levels of glucose caused different insulin secretion levels at $1240.4 \pm 109.3$ and $3539.8 \pm 245.1 \mathrm{mU} / \mathrm{L}$ with 5.5 and $25 \mathrm{mmol} / \mathrm{L}$ treatments, respectively. More importantly, the transplantation of induced cells recovered the serum glucose in Streptozotocin mice to normal levels. Coexpression of exogenous PDX-1 and NKX6.1 efficiently induced the differentiation of BMSCs into insulin-producing cells, which restored the blood glucose levels in STZ-induced diabetic mice.
\end{abstract}

Keywords: Pancreatic and duodenal homeobox factor 1, Adeno virus vector, Insulin-producing cells, Gene-modification.

\section{INTRODUCTION}

The islet transplantation is a promising approach to treat diabetes. Theoretically, successful islet transplantation can not only control the blood glucose, but also recover the physiological functions of pancreatic islets to sustain the homeostasis, which should fundamentally improve the life quality of type I diabetic patients. However, the availability of human donor for islet transplantation is always limited, thus the insulinreplacement cells produced in vitro have been considered as a critical alternative. Indeed, several studies have successfully remodeled the differentiation of embryo $\beta$ cells by inducing the formation of insulin-producing cells from bone marrow mesenchymal stem cells ${ }^{1-4}$. These promising methods, however, are still far from being applied in clinics.
Significant progress has been made towards the understanding of the molecular mechanisms of $\beta$ cell differentiation through gene knockout in mice ${ }^{5,6}$. The exocrine and endocrine cells in pancreas are originated from the precursor cells that express the pancreatic and duodenal homeobox factor 1 (PDX1), the most important and the first transcription factor in pancreatic differentiation ${ }^{4,7,8}$. PDX1 promotes the differentiation and development of stem cells to pancreatic cells then to endocrine cells, in synergy with some other transcription factors, such as Neurogenin 3 (NGN3), paired box gene 4 (Pax4) and NK6 homeobox 1 (NKX6.1) $)^{7-11}$. In more details, the endocrine precursor cells are usually determined as expressing both PDX1 and NGN $3^{4,7}$. However, without NKX6.1, the formation of $\beta$-cells becomes abnormal while other cells in pancreas are not affected ${ }^{8-10}$, demonstrating the 
critical role of NKX6.1 in $\beta$-cell differentiation. Several studies have managed to restore the expression of NKX6.1 in the PDX1-positive cells that carried NKX6.1 mutations. However, the $\beta$-cell functions were only partially recovered ${ }^{8-10}$. This suggests that PDX1 and NKX6.1 play an important role in development of $\beta$-cells. Therefore, we investigated this synergy in this study to clearly understand the mechanisms of the differentiation of BMSCs into insulin-producing cells.

\section{EXPERIMENTAL}

All restriction enzymes and T4 DNA ligase were purchased from New England Biolabs (USA); CIP (Alkaline Phosphatase, Calf Intestinal) enzyme, Endo-Free Plasmid Maxi Kit (\#D6926-B) and E.Z.N.A. Gel Extraction Kit(V-spin) (\#D2500, D2501)were purchased from omega (USA); DH5 $\alpha$ bacteria and adenovirus pAdxsi vector were purchased from SinoGenoMax (China). The pUC57-Nkx6.1 and pUC57-Pdx1 plasmids were generated and saved in our institute. Briefly, the Ins- 1 cells (pancreatic $\beta$-cell-derived or insulinomaoriginated) were grown in RPMI 1640 (GIBCO Company, Grand Island, NY, USA) with $10 \%$ fetal calf serm (FCS). The cells were incubated at $37^{\circ} \mathrm{C}$ in a humidified atmosphere of 5 $\% \mathrm{CO}_{2}$ and sub-cultured until conûuence was reached. Total RNA was prepared using the Trizol reagent following the manufacturer manual (Life Technologies, Inc). One $\mu \mathrm{g}$ of total RNA was reverse transcribed by Moloney murine leukemia virus reverse transcriptase under standard conditions. Duplicate samples of $1 \mu \mathrm{L}$ each cDNA was amplied by PCR. The amplication reaction mixture $(50 \mu \mathrm{L})$ contained cDNAs (Pdx1: forward primer 5'-AA GCTAGC CCGCAGCC $\underline{\text { ATGA-3' and }}$ reverse primer 5'-TC CTCGAG TCATCGTGGTTCCTG-3'; Nkx6.1: forward primer 5'-TA CTCGAG CGTGGGATGTTAGCGGT-3' and reverse primer 5'-TA GAATTC CGGCGT TCA GGATGAGC-3'). RT-PCR was carried out to detect the expression of the pdx 1 gene and the Nkx6.1 gene. PCR products were purified using the DNA gel extraction kit according to the manufacturer's instruction; the Pdx 1 fragment and pUC57 were digested with Nhe I and Xho I; the Nkx6.1 fragment and pUC57 were digested with Xho I and EcoR I; the target fragment was purified using the DNA gel extraction kit according to the manufacturer's instruction; the digested pUC57 and PDX1 fragment and the digested pUC57 and Nkx6.1 fragment were ligated by T4 DNA ligase, respectively. The recombinant pUC57-Nkx6.1 and pUC57$\mathrm{Pdx} 1$ plasmids were transformed into DH5 $\alpha$ cells and amplified.

The mouse anti-human PDX1/IgG (Cat\#: CABT15563MH), rabbit anti-human NKX6.1/IgG (Cat\#: DPAB32595) and mouse anti-human Insulin/IgG (Cat\#: CABT-49157MH) were all purchased from Creative biomart (USA); rabbit antihuman C-Peptide (C-P)/IgG (Cat\#: RB-130003-1000) was purchased from RayBiotech (USA); HRP antirabbit IgG (Cat\#: 18-8816-33), HRP anti-mouse IgG (Cat\#: 18-8817-33), FITC antirabbit IgG (Cat\#: 11-4839-81) were purchased from eBioscience (USA); CY5 goat anti- mouse IgG (Cat\#: 15-176-164) and CY3 goat anti-mouse IgG (Cat\#: 115-165-003) were purchased from Jackson Immuno Research (USA).
Lipofectamine 2000 was from Invitrogen (USA). The primers were synthesized by Boya Corp, Shanghai (China). RT-PCR kit was purchased from TAKARA Biotechnology (Dalian) CO., LTD. DMEM/F12 medium and B27 were purchased from GibcoInvitrogen (USA). Fetal bovine serum was from Hyclone (USA). The epidermal growth factor (EGF), basic fibroblast growth factor (bFGF), hepatocyte growth factor (HGF), human betacellulin, glucagon-like peptide-1 (GLP-1) and nicotinamide (NIC) were all purchased from Pepro Tech (USA). Insulin direct chemiluminescence assay kit (Cat\#: 02230141) and C-peptied direct chemiluminescence assay kit (Cat\#: 03649928) were Purchased from SIEMENS (USA). The contents of insulin (measure range: $0.5 \mathrm{mU} / \mathrm{L}-300 \mathrm{mU} / \mathrm{L}$ ) and C-Peptide (measure range: $0.05 \mu \mathrm{g} / \mathrm{L}-\mu \mathrm{g} / \mathrm{L}$ ) were detected with ADVIA Centaur (Germany).

$\mathrm{BALB} / \mathrm{c}$ mice used in this study were obtained from Guangdong animal center. All mouse experiments were performed under the approval from the Ethics Committee of Guangzhou University of Chinese Medicine.

Vector construction: For the construction of pShuttleGFP-CMV-Nkx6.1 plasmid, the Nkx6.1 cDNA fragment was retrieved from pUC57-Nkx6.1 by Bgl II and EcoR I doubleenzyme digestion. Then, the fragment of interest $(1.12 \mathrm{~kb})$ was first separated by agarose gel electrophores, at the same time, the pShuttle-GFP-CMV plasmid was digested with the same enzymes and separated by gel electrophoresis. DNA ligation was carried out by incubating in the $15 \mu \mathrm{L}$ reaction solution containing $1.25 \mu \mathrm{L}(80 \mathrm{ng} / \mu \mathrm{L})$ of the digested pShuttleGFP-CMV plasmid and the digested Nkx6.1 cDNA fragment, $1.5 \mu \mathrm{L}$ of $10 \times \mathrm{T} 4$ buffer and $0.5 \mu \mathrm{L}$ T4 DNA ligase at $16^{\circ} \mathrm{C}$ overnight. The recombinant vector pShuttle-GFP-CMVNkx6.1 was transformed into DH5 $\alpha$ competent cells.

For the construction of pShuttle-CMV-Pdx1/CMV-Nkx6.1 plasmid, the Pdx1 cDNA fragment was retrieved from pUC57Pdx 1 by enzyme Xho I digestion and next by enzyme klenow digestion for $24 \mathrm{~h}$, which was then purified using the DNA gel extraction kit according to the manufacturer's instruction. Then the product Pdx1 fragment was digested with Nhe I and the fragment of interest $(0.87 \mathrm{~kb})$ was separated by agarose gel electrophoresis. The pShuttle-GFP-CMV-Nkx6.1 plasmid was digested with Nhe I and Pme I enzyme; the fragment of interest $(5.7 \mathrm{~kb})$ was separated by agarose gel electrophores; and the DNA ligation was carried out by incubating in the $15 \mu \mathrm{L}$ reaction solution containing $1.25 \mu \mathrm{L}(80 \mathrm{ng} / \mu \mathrm{L})$ of the digested pShuttleGFP-CMV-Nkx6.1 plasmid and the digested Pdx1 fragment at $16{ }^{\circ} \mathrm{C}$ overnight. The recombinant vector pShuttle-CMVPdx1/CMV-Nkx6.1 plasmid was transformed into DH5 $\alpha$ cells and amplified. The pShuttle-CMV-Pdx1/CMV-Nkx6.1 plasmid was purified using the DNA gel extraction kit according to the manufacturer's instruction and identified by $1 \%$ agarose gel electrophoresis.

For the construction of pAdxsi-CMV-Pdx 1/CMV-Nkx6.1 plasmid, pAdxsi plasmid and pShuttle-CMV-Pdx1/CMVNkx6.1 plasmid were digested with I-Ceu I and I-Sce I enzymes and then dephosphorylated, which were purified using the DNA gel extraction kit according to the manufacturer's instruction. The DNA ligation was carried out by incubating in the $15 \mu \mathrm{L}$ reaction solution containing $1.25 \mu \mathrm{L}(80 \mathrm{ng} / \mu \mathrm{L})$ 
of the digested pAdxsi plasmid and pShuttle-CMV-Pdx1/ CMV-Nkx6.1 plasmid at $16^{\circ} \mathrm{C}$ overnight, before being ligated with the inserted fragment of interest to generate the pAdxsiCMV-Pdx1/CMV-Nkx6.1 viral plasmid. This plasmid was also amplified in DH5 $\alpha$ cells. The recombinant vector was transformed into DH5 $\alpha$ competent cells and amplified. The confirmed plasmids were extracted by plasmid DNA minipreparation kit according to the manufacturer's instruction and identified by $1 \%$ agarose gel electrophoresis. The plasmid concentrations were evaluated with an ultraviolet spectrophotometer.

Virus production and titer determination: The HEK 293 cells (ATCC, Manassas, VA, USA) were cultured in DMEM supplemented with $10 \% \mathrm{FBS}$ at $37^{\circ} \mathrm{C}$ with $5 \% \mathrm{CO}_{2}$. Lipofectamine 2000 was used for the transfection of pAdxsiCMV-Pdx1/CMV-Nkx6.1, which was digested with Pac I enzyme, into 293 cells at $80 \%$ confluency. The cell supernatant with virus was collected 3-5 days after transfection when most of the cells showed morphological changes and plaques. The viral solution was saved at $-80^{\circ} \mathrm{C}$. The titer of virus was determined using 293 cells following the AdEasy Vector Systerm method. In brief, HEK293 cells were seeded onto 24-well plates and grown to $90 \%$ confluency, before being infected with a series of virus dilutions (ranged from $10^{1}-10^{12}$ ) until plaque formation was observed. The virus titer (plaque forming unit $(\mathrm{PFU}) / \mathrm{mL}$ ) was calculated by the following formula: Virus titer $=($ number of plaques $\times$ dilution $) /$ volume of diluted virus added to the well.

Culture and isolation of bone marrow mesenchymal stem cells: Bone marrow (BM) samples were obtained from healthy donors (35-45 years of age) with informed consents. The heparin anticoagulated bone marrow was mixed with 3$4 \mathrm{~mL}$ PBS and the mononuclear cells in this mixture were isolated by gradient centrifugation with $1.077 \mathrm{~g} / \mathrm{mL}$ percoll centrifugal separation liquid. The isolated cells were then seeded into plastic flask for culture. Besides the mononuclear cells as the major component, the isolated cells also contained some macrophages and monocytes that were removed through medium change and subsequent passaging. The purified mononuclear cells were seeded into $75 \mathrm{~cm}^{2}$ flasks containing FM, Dulbecco's-modified Eagle's Medium-Low Glucose (DMEM-LG; Gibco/Invitrogen, Carlsbad, CA) with $10 \%$ fetal calf serum (FCS; Hyclone, Logan, UT) and cultured at $37{ }^{\circ} \mathrm{C}$ in $5 \% \mathrm{CO}_{2}$. The medium was removed $48 \mathrm{~h}$ later by washing with PBS and was subsequently changed every 3-4 days. As the cells grow to 80-90\% confluency, the MSCs were recovered using $0.25 \%$ Trypsin- $0.02 \%$ EDTA and replated at 1:3. Flow cytometry was used to analyze the phenotypes and the labeling markers of BMSCs after three passages.

Virus infection and exogenous gene expression: The hMSCs (third and fourth generations) were seeded into 75 $\mathrm{cm}^{2}$ flasks at a density of $5 \times 10^{6}$ cells/flask. When reaching $90 \%$ confluency, the cells were divided into three groups: the first group was infected with the Adxsi-CMV-Pdx1/CMVNkx6.1 virus (experimental group, EG); the second goup of cells were infected with the empty-vector virus (viral control group, CVG), Virus added to the culture flask with 100CFU/ cell and the third group of cells were the BMSCs control group without infection (control group, CG). After 48 h, fluorescence microscopy results show more than $95 \%$ of the cells infected with the virus. Three group cells were washed in PBS and recovered using trypsin. RT-PCR was carried out to detect the expression of the genes of interest. The following primers were used: Pdx1 (F: 5'-ACC TTC ACC ACC ACC TCC CG-3' and R: 5'-TTC AAC ATG ACA GCC AGC TCC AC-3', annealing at $61^{\circ} \mathrm{C}$ and $310 \mathrm{bp}$ PCR product); Nkx6.1 (F: 5'-CAA TGG AAG GCA CCA GAC A-3' and R: 5'-GCT ACG GGC ATA GAG GGT C-3', annealing at $57^{\circ} \mathrm{C}$ and $313 \mathrm{bp}$ PCR product); GAPDH (F; 5'-GTC AGT GGT GGA CCT GAC CT-3' and R: 5'-TGA GGA GGG GAG ATT CAG TG-3', GAPDH was used as an internal control to normalize variable amounts of cDNA in each sample and 400 bp PCR product); Ngn3 (F: 5'-AAA GCG AGT TGG CAC TAA GCA-3' and R: 5'-CGT CTG GGA AGG TGG GAA GTA-3', annealing at $61^{\circ} \mathrm{C}$ and 132 bp PCR product); Insulin (F: 5'-AGC CTT TGT GAA CCA ACA CC3' and R: 5'-GCT GGT AGA GGG AGC AGA TG-3', annealing at $65^{\circ} \mathrm{C}$ and $245 \mathrm{bp}$ PCR product); and GLUT2 (F: 5'-AGG ACTT CTG TGG ACC TTA TGT G-3' and R: 5'-GTT CAT GTC AAA AAG CAG GG-3', annealing at $55^{\circ} \mathrm{C}$ and 231 bp PCR product).

Cytokine induction: Four different treatments were given to the BMSCs: the 0-stage treatment with which the normal cells received no infection and chemical treatment; the I-stage treatment with which the cells were infected with Adxsi-CMVGFP and Adxsi-CMV-Pdx1/CMV-Nkx6.1 viruses for three days; the II-stage treatment with which the cells were incubated with epidermal growth factor (EGF) at $80 \mathrm{ng} / \mathrm{mL}$ and basic fibroblast growth factor (bFGF) at $10 \mathrm{ng} / \mathrm{mL}, \mathrm{B} 272 \%$ in DMEM/F12 medium containing $5 \%$ FBS for four days; and the III-stage treatment with which the cells were cultured in glucagon-like peptide-1 (GLP-1) at $10 \mathrm{ng} / \mathrm{mL}$, human betacellulin at $10 \mathrm{ng} / \mathrm{mL}$, hepatocyte growth factor (HGF) at $10 \mathrm{ng} / \mathrm{mL}$, nicotinamide (NIC) at $10 \mathrm{mmol} / \mathrm{L}, \mathrm{B} 27$ at $2 \%$ and $\beta$-mercaptoethanol at $0.1 \mathrm{mmol} / \mathrm{L}$ in DMEM/F12 medium containing $5 \%$ FBS for seven days.

Cell immunophenotyping and in vitro multilineage differentiation studies: About $5.0 \times 10^{5}$ cells were trypsinized to obtain cell suspension $(5 \mathrm{~mL})$, then incubated $0.5 \mathrm{~h}$ in the dark at $25^{\circ} \mathrm{C}$ with $10 \mu \mathrm{L}$ of fluorochrome-conjugated (FITC or PE) monoclonal antibody (BD Pharmingen and MACS). Cells were analyzed for haematopoietic (HLA-DR, CD19, CD34 and CD45) and mesenchymal (CD106, CD29 and CD44) markers at different passages (P3, P5 and P7) to define the purity. After incubation with the specific antibody, cells were washed and analyzed with a FACS-caliber instrument (BD FACSCalibur, Germany).

Adipogenesis, chondrogenesis and osteogenesis for BMSCs were carried out in the appropriate induction media according to the manufacturer's protocol(Cambrex, MD http:// www.cambrex.com). The differentiation phenotype was documented using oil red-O for adipocytes and Alizarin staining for osteocytes. Dithizone (DTZ) stain of $10 \mathrm{mg} / \mathrm{mL}$ in DMSO (dimethyl sulphoxide) concentration used to stain islet like cell aggregates.

Dithizone staining and immuno-staining: $0.1 \mathrm{~mL}$ of the stock solution containing dithizon (DTZ) (50 mg DTZ dissolved 
in $5 \mathrm{~mL}$ DMSO) was added into $10 \mathrm{~mL}$ PBS for dithizone staining at $37^{\circ} \mathrm{C}$ for $15 \mathrm{~min}$. The stained cells were examined under microscope.

After 3, 7 and 14 days transfection, the cells were seeded on glass cover slides $(20 \mathrm{~cm} \times 20 \mathrm{~cm})$ for immuno-staining. Before being incubated with primary antibodies, the cells were fixed with $4 \%$ paraformaldehyde (PFA) in PBS for $20 \mathrm{~min}$, permeabilized using $0.3 \%$ Triton $\mathrm{X}-100$ for $10 \mathrm{~min}$ and blocked with $10 \%$ lamb serum and $5 \%$ bovine serum albumin (BSA) for $0.5 \mathrm{~h}$. The antibodes used were monoclonal mouse anti-human/mouse PDX1, polyclonal rabbit anti-human insulin and monoclonal mouse antihuman $\mathrm{C}$-peptide. The primary antibody-inculated cells were then inculated: with the horse radish peroxidase (HRP)-conjugated antimouse secondary antibody for the 3,32-diaminobenzidine (DAB) staining to visualize the Insulin and C-peptide in cytoplasm and the PDX1 and NKX6.1 in nucleus; or with FITC- conjugated sheep antirabbit IgG or CY5- conjugated sheep antimouse IgG for $0.5 \mathrm{~h}$ for indirect fluorescence detection.

Determination of insulin and C-peptide secretion: The cells seeded in 6-well plates of control group, empty virus control group and experimental group with each stage, approximately $1 \times 10^{5}$ cells/well, washed 2 times with PBS, respectively, then were treated with the $1 \mathrm{~mL}$ of PBS buffer containing $5.6 \mathrm{mmol} / \mathrm{L}$ or $25 \mathrm{mmol} / \mathrm{L}$ glucose incubation for $2 \mathrm{~h}$. After the treatment, the supernatant was collected to detect the levels of insulin and C-peptide by the chemiluminescence methed by following the manufacturer's instruction.

Western blot analysis: To detect the expression level of transcription factor and insulin molecule in the experimental group at different stages, cells were treated with lysis buffer and nuclear and cytoplasmic proteins were extracted in the corresponding phase by following the manufacturer's instruction.

Protein concentrations were determined using the PIERCE BCA protein assay kit. Proteins were separated by $10 \%$ SDSPAGE under denaturing conditions and transferred to nitrocellulose membranes. Membranes were incubated with mouse anti human monoclonal antibody (1:1,000; Santa Cruz Biotechnology), followed by incubation in goat antimouse secondary antibody conjugated with horseradish peroxidase (1:1,000-1,500; Santa Cruz Biotechnology). Immunoreactive proteins were detected with the enhanced chemiluminescence detection system (Amersham Biosciences).

Effects of the induced cells on hyperglycemia in diabetic mice: To determine whether the Adxsi-CMV-Pdx1/CMVNkx6.1 BMSC cell-derived islet-like cells corrected the hyperglycemia in diabetic mice, pathogen-free pure-bred BALB/c mice (25-30 g of weight, 6-week of age) were purchased from Guangdong Laboratory Animal Research Center for this study. Hyperglycemia was generated in the mice were by i.p. injection of streptozotocin (STZ) at $75 \mathrm{mg} / \mathrm{kg}$ of body weight and the blood glucose was detected with an Accu-Chek Aviva Glucose Meter (Electrochemical assay, Roche). When the blood glucose levels reached $22.2 \mathrm{mmol} / \mathrm{L}$ and higher, the mice were identified as being diabetic. Before implantation, diabetes was confirmed by the presence of blood glucose at concentrations higher than $22.2 \mathrm{mmol} / \mathrm{L}$. The mice were then randomly divided into the STZ control group, the STZ BMSCs control group, the STZ empty vector control group and the STZAdxsiCMV-Pdx1/CMV-Nkx6.1 experimental group.

For cell implantation, $1 \times 10^{6} \mathrm{BMSC}$ or islet-like cells were transplanted under the kidney capsule in STZ-induced diabetic mice. Every 2 days, blood was collected from the snipped tail of mice to measure blood glycose. All experimental procedures involving animals were approved by the Ethics Committee of Guangzhou University of Chinese Medicine and performed in accordance with the guidelines set by the China Animal Care and Use Guidelines.

Statistical analysis: All data are presented as the mean \pm SEM. Multivariate analysis of variance was used to determine the statistical significance among different treatments in each group, A probability $(p)$ value $<0.01$ or 0.05 was considered to be significant.

\section{RESULTS AND DISCUSSION}

Verification of plasmid construction: As shown in Fig. 1, digestion of pShuttle-GFP-CMV-Nkx6.1 by Bgl II and EcoR I resulted in two bands at 1.12 and $5.1 \mathrm{~kb}$. The digestion of pShuttle-CMV-Pdx1/CMV-Nkx6.1 by Xho I resulted in two bands at 1.8 and $4.6 \mathrm{~kb}$ and the digestion of pAdxsi-CMVPdx1/CMV-Nkx6.1 by Xho I brought 7 bands on the gel: 14, $11.8,3.9,2.66,2.47,1.45$ and $0.6 \mathrm{~kb}$. The enzyme digestion results were all as expected, demonstrating the successful construction of desired plasmids.
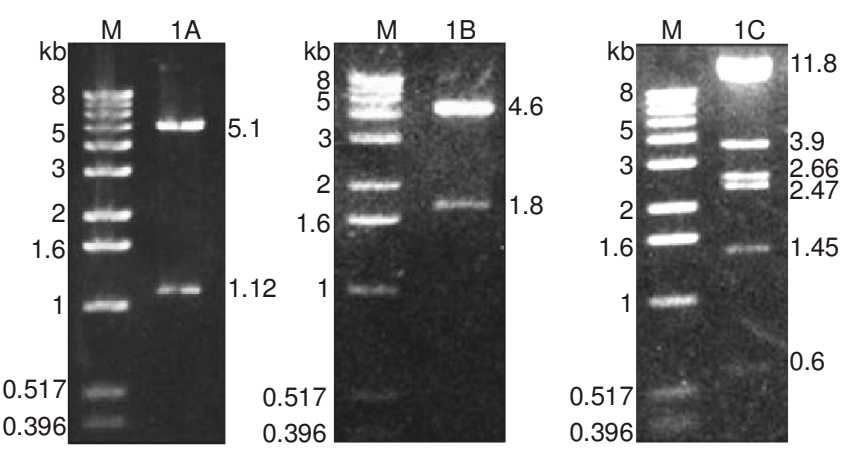

Fig. 1. Successful plasmid construction. 1A and 1B: 4 bands showed the right sizes after digestion with $\mathrm{Bgl}$ II and EcoR I enzymes from pShuttle-GFP-CMV-Nkx6.1. 1C: 8 bands showed the right sizes after digestion with Xho I enzyme from pAdxsi-CMV-Pdx1/CMVNkx6.1. M: Marker: 8 kb, 7 kb, 6 kb,5 kb,4 kb,3 kb,2 kb,1.6 kb,1 $\mathrm{kb}, 0.517 \mathrm{bp} 0.396 \mathrm{bp}$; $1 \mathrm{~A}$ : The digested fragments of pShuttleGFP-CMV-Nkx6.1 plasmid by EcoR I and Bgl II; 1B: The digested fragments of pShuttle-CMV-Pdx1/CMV-Nkx6.1 plasmid by EcoR I and Bgl II; 1C: The digested fragments of pAdxsi-CMV-Pdx1/ CMV-Nkx6.1 plasmid by Xho I

Expression patterns of CD106, CD44 and CD29 in BMSCs during multilineage differentiation: FACS analysis revealed that BMSCs at different passages (P3, P5 and P7) had the same surface markers of mesenchymal stem cells. The cells showed simultaneous expression of cell surface mesenchymal markers, including CD106, CD44 and CD29 (> $98 \%$ of cells were positive) with a concomitant absence of markers, HLA-DR, CD34, CD45 and CD19 ( $<5 \%$ of cells were positive). At the same time, BMSCs exhibited in vitro competence to differentiate into adipogenic and osteogenic lineages upon 
specific induction, as being confirmed by the Oil red-O staining and the Alizarin-red staining, respectively.

Changes in morphology and protein expression: After the second passage, the BMSCs started to exhibit their characteristic spindle-shaped morphology (Fig. 2A). After Adxsi infection $48 \mathrm{~h}$, fluorescence microscopy results show more than $95 \%$ of the cells infected with the virus. $24 \mathrm{~h}$ later, the BMSCs in the experimental group infeced Adxsi-CMVPdx1/CMV-Nkx6.1 began to express the transcription factors PDX1 and NKX6.1 that were mainly observed in the nucleus (Fig. 2B,C). The morphology of BMSCs in the experimental group began to change after the induction of PDX1 and NKX6.1 together with EGF, bFGF and B27. The cell shape turned round and the cells gathered gradually to form pancreatic islets (Fig. 2D), but the morphology of BMSCs in control groups keep spindle-shaped morphology.

Under microscope, the cytoplasm was revealed by the dithizone staining as shiny red (Fig. 2E). Immunocytochemical staining revealed that PDX1 was mainly expressed in the nucleus (Fig. 2F), although the insulin was observed in intracytoplasm (Fig. 2G). Indirect fluorescence staining demonstrated that PDX1 (CY5) and NKX6.1 (FITC) were both expressed in the nucleus (Fig. 2H). The expression of insulin (CY5) was present (Fig. 2J,2L) in the cells with II-stage treatment and the Cpeptide (FITC) was expressed in the III-stage cells (Fig. 2K,L). No significant morphological changes were observed in the cells without any treatment and no insulin or C-peptide expression.

Changes in the expression of related genes: AdxsiCMV-Pdx1/CMV-Nkx6.1 infected BMSCs expressed PDX1 and NKX6.1 from $24 \mathrm{~h}$ to 3 weeks after infection. The expression of the $\beta$-cell related factor NGN3 was in synergy with PDX1 and NKX6.1. The insulin expression was promoted by the II-stage treatment of cytokine EGF and bFG.F The insulin secretion was higher in III-stage, together with higher expression of the $\beta$-cell related factor Glut 2 and high level of glucose, while the expression of PDX1 and NKX6.1 was kept stable in this stage (Fig. 3). These expression patterns were verified by western blot analyses (Fig. 4).

Determination of insulin and C-peptide levels by chemoluminescence: The basal insulin level was maintained at $(4.1 \pm 4.7) \mathrm{mU} / \mathrm{L}$. In the experimental group, the insulin levels reached $5.3 \pm 5.7,334.1 \pm 45.6$ and $1240.4 \pm 109.3$ $\mathrm{mU} / \mathrm{L}$ in the I-, II- and III-stages, respectively (Fig. 5A). With the hyperglycemic stimulus, the insulin level became even higher at $3539.8 \pm 245.1 \mathrm{mU} / \mathrm{L}$. The insulin levels of the BMSCs control group did not show significant differences between the II-stage treatment and the III-stage treatment.

As shown in Fig. 5, the C-peptide was only detected in the experimental group. It started to express from the end of the II-stage at $0.063 \pm 0.07 \mu \mathrm{g} / \mathrm{L}$ and became $1.42 \pm 0.21 \mu \mathrm{g} / \mathrm{L}$ in the III-stage. The C-peptide level was significantly increased to $3.57 \pm 0.62 \mu \mathrm{g} / \mathrm{L}$ with hyperglycemic stimulation. The results of $\mathrm{C}$-peptide and insulin indicate that the cells in the experimental group were able to secret insulin, just like the pancreatic $\beta$-cells.

Effects of induced cells on diabetic mice: The basal level of blood glucose in a normal BALB/c mouse was about $5.0 \pm$ $0.9 \mathrm{mmol} / \mathrm{L}$, but the diabetic mice had $25.4 \pm 2.7 \mathrm{mmol} / \mathrm{L}$ of
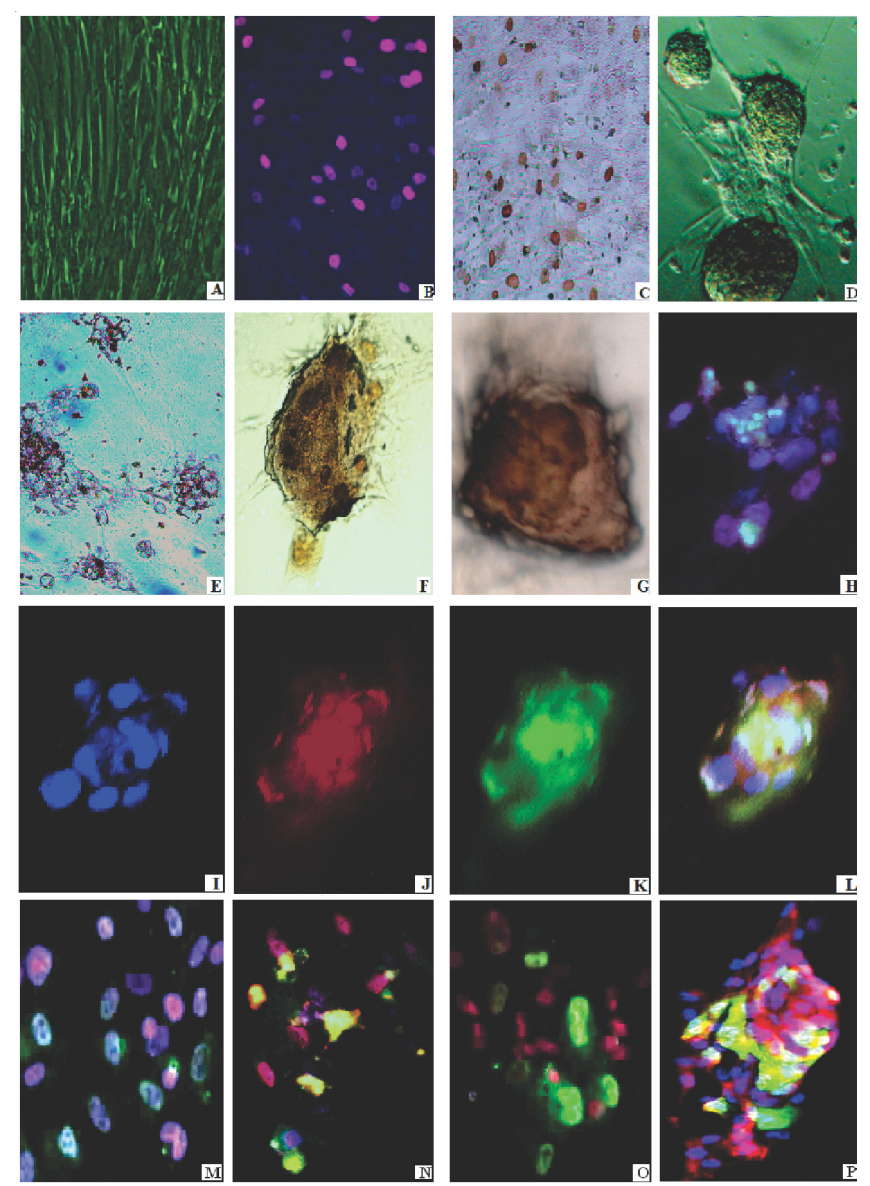

Fig. 2. Changes in morphology and protein expression in the infected BMSCs. Results of immumofluorescence or immunohistochemistry for the infected cells in different stages. A: BMSCs of passage 3, $\times 200$; B, C: the expression of PDX1 (B) and NKX6.1 (C) in experimental group, after Adxsi-CMV-Pdx1/CMV-Nkx6.1 infected for 7 days $\times 200$; D: After Adxsi-CMV-Pdx 1/CMV-Nkx6.1 infection, experimental cells were exposed to serum free media for 2 days, cells migrated and clustered to become islet-like cells aggregates; E: Dithizone staining of islet like cells aggregates were positive $\times 200$; F: Immunocytochemistry staining of islet like cells aggregates were PDX1 postive $\times 200$; G: Immunocytochemistry staining of islet like cells aggregates were insulin postive $\times 200$; $\mathrm{H}$ : The nuclei of the cells were stained blue with DAPI (4', 6-diamidoino-2phenylindole), PDX1(CY5) and NKX6.1 (FITC) expression in the nucleus of islet-like cell aggregates, $\times 400$; I-L: insulin (CY5) and C-P (FITC) expression in islet-like cells 11 days after infection, $\times 400$; M: staining of cells by immune method might give false positive results. The thin slides of islet-like cells were prepared and staining was repeated with indirect fluorescence to indicate the expression of PDX1 (CY5) and NKX6.1 (FITC) in the nuclei ( $\times$ 400); N: PDX1(CY5) and MafA (FITC) expression in the nuclei ( $\times$ 400); O: PDX1 (CY5) and MafB (FITC) expression in the nuclei of different cells $(\times 400)$; P: Insulin/Proinsulin (CY3) and glucagon(FITC) expression in the cytoplasm of the islet-like body of the experimental group $(\times 200)$

blood glucose levels. As shown in Fig. 6. The control diabetic animals that were transplanted with empty-vector infected cells or BMSCs (with or without GLP-1 treatment) showed temporarily decreasing blood glucose levels, before returning to the original blood glucose levels 4 days later. However, the glucose levels of diabetic mice in the treatment group $(n=30)$ that were transplanted with the differentiated islet-like cells were able to restore near mornoglycemia within 3-4 weeks; the glucose level of the treatment group was at $6.9 \pm 2.3 \mathrm{mmol} / \mathrm{L}$ 

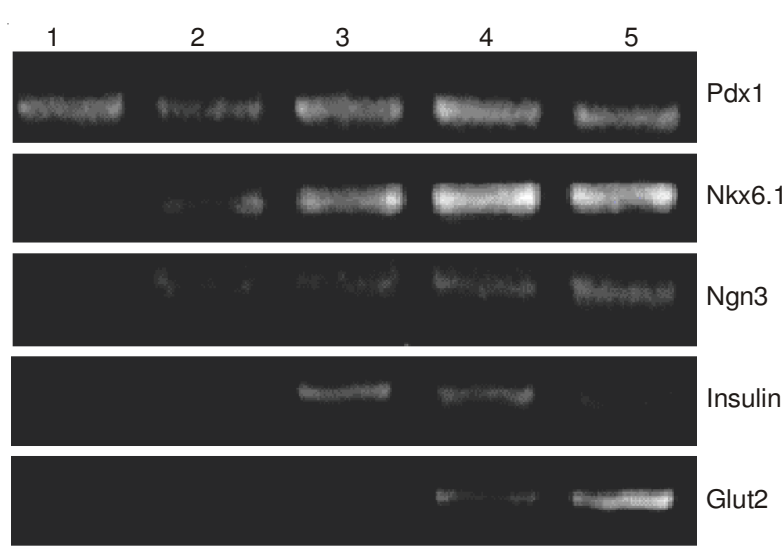

Fig. 3. Expression of Pdx1, Nkx6.1, Ngn3, Insulin and Glut2 in the experimental cells at different stage. RT-PCR products visualized on agarose gels. 1: $24 \mathrm{~h}$ after Adxsi-CMV-Pdx1/CMV-Nkx6.1 infection, only Pdx1 mRNA was positive; 2-5: After Adxsi-CMVPdx1/CMV-Nkx6.1 infection, the expression of Pdx1, Nkx6.1, Ngn3, Insulin and GLUT2 in the experimental cells at the 3th,7th,14th and 14th day with high glucose levels. Ngn3 began to be expressed on the 3rd day; Insulin began to express on the7th day; and Glut2 only began to be expressed on the 14th day; but on the 14th day, when the experimental cells were treated with high glucose levels $(25 \mathrm{mmol} / \mathrm{L})$, the experimental cells expressed Pdx1, Nkx6.1, Ngn3, Insulin and Glut2

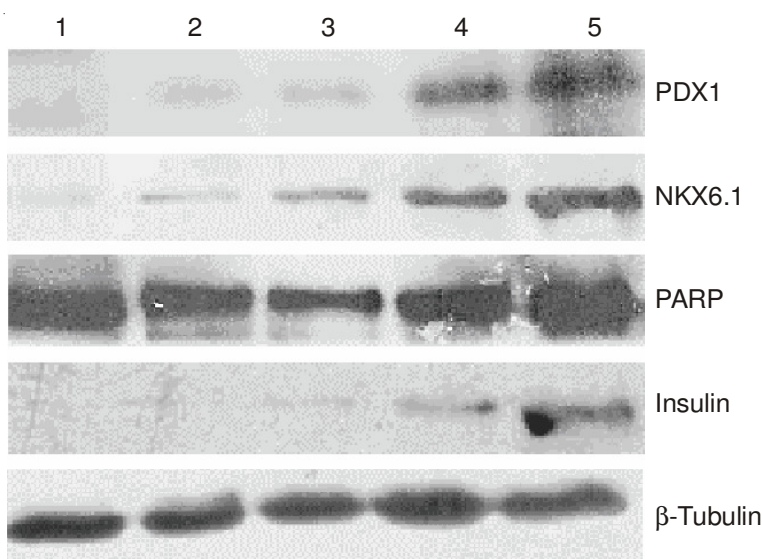

Fig. 4. Protein expression of PDX1 and NKX6.1 and insulin. Results from western-blot analyses. 1: BMSCs before infection, cells did not express PDX1, NKX6.1 and insulin; 2-5: PDX1, NKX6.1 and insulin expression in cells induced by Adxsi-CMV-Pdx1/CMVNkx6.1 at the 2th, 7th, 14th day and 14th days with high glucose levels. After being induced for 7 days, the experimental cells induced by Adxsi-CMV-Pdx1/CMV-Nkx6.1 began to express insulin. After 14 days, insulin level in experiment cells was much higher than that treated with low glucose $(5.6 \mathrm{mmol} / \mathrm{L})$ when the experimental cells at stage III were treated with high glycose concentration ( 25 $\mathrm{mmol} / \mathrm{L}$ ) for $2 \mathrm{~h}$

in 2 days and at $6.5 \pm 1.5 \mathrm{mmol} / \mathrm{L}$ in 32 days after cell transplantation, which was much lower than that of the STZ control group $(n=20)$, that of the STZ BMSCs control group $(n=30)$ and that of the STZ empty vector control group $(n=30)$. The blood glucose levels in STZ-induced diabetic nude mice showed significant decreases and the mortality of treatment group also decreased compared with the STZ control group and STZ BMSCs group. After transplant, the blood glucose level of the treatment group was maintained at $<6.9 \mathrm{mmol} / \mathrm{L}$ in $95 \%$ of the experimental mice and the average survival time was 30 days.
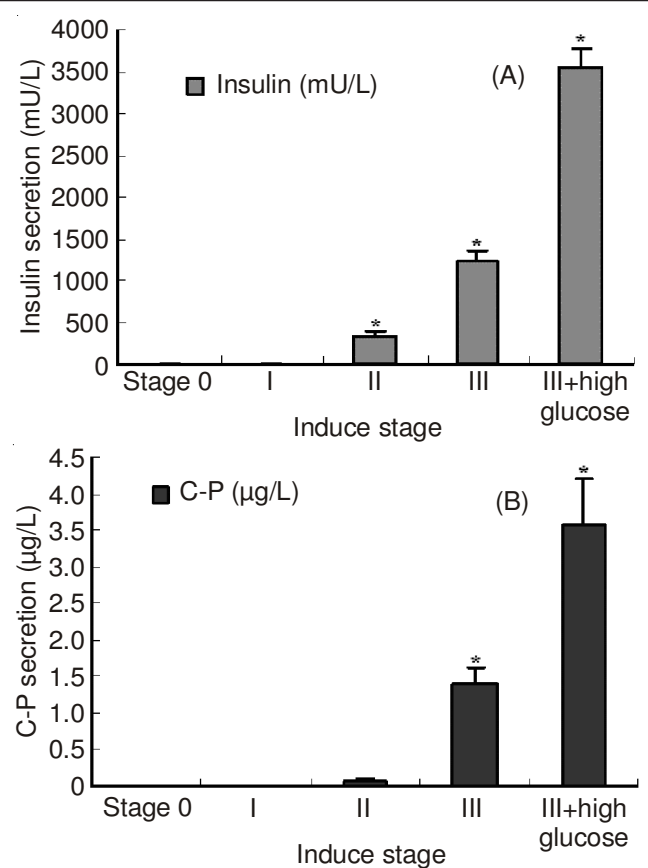

Fig. 5. Secretion of Insulin and C-peptide from BMSCs after being infected with Adxsi-CMV-Pdx1/CMV-Nkx6.1 in different induced stages that were determined by chemoluminescence assay $(p<0.05, \mathrm{n}=$ 4). Expremental cell supernatants were collected and analyzed for insulin and C-peptide release by the chemiluminescence methed. At stages 0 and I, expremental cell supernatant did not have Insulin or C-peptide; at stage II, lower level of Insulin and C-peptide were measured; at stage III treated with low glucose concentration $(5.6 \mathrm{mmol} / \mathrm{L})$; Insulin or C-peptide contents were much higher than that at stage II and when cells at stage III treated with high glycose concentration $(25 \mathrm{mmol} / \mathrm{L})$; Insulin or C-peptide content were much higher than that at stage III treated with low glucose concentration $(5.6 \mathrm{mmol} / \mathrm{L})$

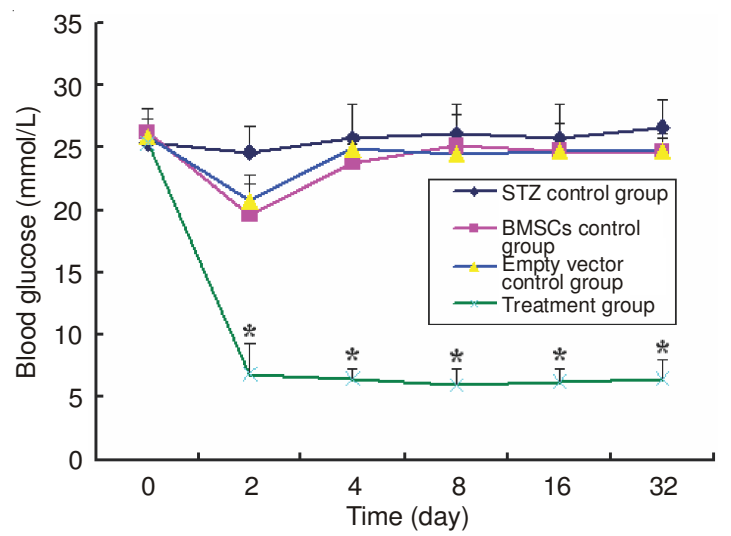

Fig. 6. Production and secretion of insulin by differentiated cells in murine diabetes model. BALB/c mice of the STZ control group $(n=20)$ were treated with STZ to induce diabetes and hyperglycemia and glucose levels at the indicated time points after transplantation are given as means \pm SDs. Diabetes did not regress in response to the transplantation of BMSCs (the STZ BMSCs control group, $\mathrm{n}=30$ ) or that of BMSCs infected empty vector (the STZ empty vector group, $\mathrm{n}=30$ ) under the kidney capsule of STZ-induced diabetic mice. Diabetic mice that were transplanted with undifferentiated BMSCs developed more severe hyperglycemia in the weeks following the transplant. However, the glucose levels of diabetic mice of the treatment group that were transplanted with the differentiated islet-like cells could restore near mornoglycemia within 3-4 weeks; the glycose level of the treatment group $(n=30)$ was much lower that of the STZ control group and those of the STZ BMSCs control group and the STZ empty vector control group $(p<0.001)$ 
Characteristics of transplanted cells: In order to detect the secreting activity of the transplanted cells, the mice were sacrificed on the 35 th day after transplantation. The diabetic mice in the STZ control group exhibited elevated blood glucose levels ( $>25 \mathrm{mmol} / \mathrm{L}$ ), polyuria, muscle wasting, weight loss, poor mobility, shivering and died within 18-33 days. Blood glucose of the STZ BMSCs control group and the STZ empty vector control group diabetic mice had decreased slightly during 4 days of post-BMSCs transplantation, then these mice continued to exhibit hyperglycemia to died. The STZ AdxsiCMV-Pdx1/CMV-Nkx6.1 experimental group showed remarkable correction of the hyperglycemia as early as Day 2 posttransplantation. Further, these mice continued to exhibit near normoglycemic levels beyond 33 days post-transplantation (Fig. 6).

To confirm the function of the Adxsi-CMV-Pdx1/CMVNkx6.1 BMSC cell-derived islet-like cells in the kidney, the kidneys with transplanted cells (BMSCs or islet-like cells) were fixed in $4 \%$ PFA for histopathological, immunohistochemical and indirect fluorescence staining. HE staining showed that normal renal tissue-wrapped renal capsule, nephron and renal structure were clear (Fig. 7A). In the STZ BMSCs control group and the STZ empty vector control group (Fig. 7B), fusiform BMSCs were under the renal capsule; the islet-like cells of the experimental group were round or oval, larger in cell size, abundant cytosol and basophilic with secretory vacuole formation and the nucleus to one side, suggesting active endocrine function (Fig. 7C).

Insulin immunohistochemical staining of renal capsule and cortex were negative, with a large number of insulin positive cells in the experimental group's kidney subcapsular (Fig. 7D). The renal cortical cells adjacent to islet-like cells in the experimental group were positive, possibly due to the insulin released into the blood circulation system via the renal cortex in the experimental group. Further examination of the experimental group and the other control groups, the expression and localization of NGN3 and MafA transcription factors in transplanted cells, the transplanted cell nuclei in the control groups were negative, but part of the islet-like cell nuclei in the experimental group were positive (Fig. 7E,F). Taken together, our results highlight the potential of the Adxsi-CMVPdx1/CMV-Nkx6.1 inducting BMSCs to generate islet-like cells that can be used therapeutically. Finally, to rule out the possibility of endogenous pancreatic $\beta$ cell regeneration, we performed hematoxylin-eosin staining and immunohistochemistry of the pancreas on day 32 from the STZ treated mice that were transplanted with islet-like cells. Histopathology (Fig. 7G) demonstrates that the normal pancreas has large pancreatic islets. However, the pancreas from the mice transplanted with islet-like cells revealed near complete loss of pancreatic islets without evidence of endogenous pancreatic $\beta$-cell regeneration (Fig. $7 \mathrm{H}$ ) and part of destructed islet was instead inflammatory cells (Fig. 7I).

Ever since the mesenchymal stem cells were successfully induced to form islet-like cells in vitro, this new technology has brought so much hope to diabetes therapy ${ }^{1}$. However, the insulin secretion of induced pancreatic islet cells has always been much less efficient than the normal islet cells ${ }^{1,2,4-9}$. Therefore, a new therapy will not become reality until the insulin

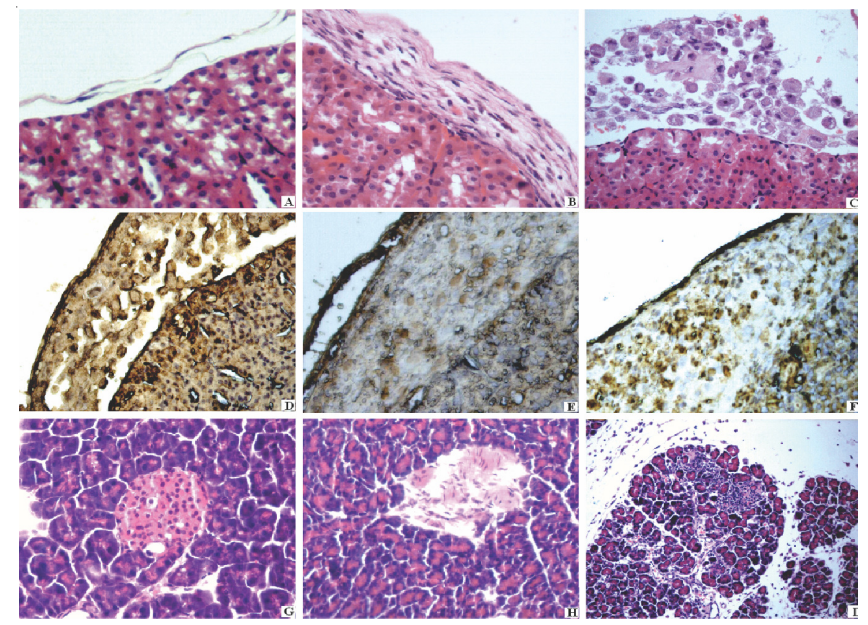

Fig. 7. Experimental cells of different group were transplanted under the kidney capsule of streptozotocin-induced diabetic mice. 33 days after transplantation, the kidneys were removed and sectioned. HE staining of normal kidney and kidney capsule structure $(\mathrm{A}, \times 200)$. BMSCs of the STZ BMSCs control group and the STZ empty vector control group $(\mathrm{B}, \times 200)$ were transplanted under the kidney capsule; BMSCs showed no changes in cell morphology and cells were fusiforms. However, the Pdx1/Nkx6.1-derived islet-like cells (stage 3 ) were transplanted under the kidney capsule of diabetic mice and the islet-like cells (stage 3) were round or oval, abundant in cytoplasm and basophils with secretion of vacuolization and nuclei pressed against the side of the prompt secretion of functionally active $(C, \times 200)$, indicating that the cellular secretory function was active. The islet-like cells were immunopositive for Insulin (D, $\times 200)$, MafA $(\mathrm{E}, \times 200)$ and NGN3 $(\mathrm{F}, \times 200)$. Histopathology demonstrated that the normal pancreas had large pancreatic islets $(\mathrm{G}, \times 200)$. However, the pancreas from the mice transplanted with islet-like cells revealed near complete loss of pancreatic islets and no evidence of endogenous pancreatic $\beta$-cell regeneration $(\mathrm{H}, \times 200)$ ) and part of destructed islet was instead of inflammatory cells (I, $\times 100$ )

secretion efficiency of the induced islet cells is significantly improved. In this study, we managed to induce islet-like cells by exogenously expressing both PDX1 and NKX6.1, along with the treatment of cytokines, especially Glucagon-like peptide-1 (GLP-1). The islet-like cells obtained in this study had increased insulin synthesis and secretion, which were also able to recover the serum normal glucose levels in the diabetic mice.

The gene expression regulation of insulin in pancreatic $\beta$-cells is extremely complicated ${ }^{8,10,11}$. For example, the promoter of the insulin gene contains abundant cis-acting elements that bind to multiple transcription factors, such as A box (A2, A3), E box (E1, E2) and C2 and C1/RIPE3b ${ }^{12-16}$. The $\beta$ cell-specific transcription factor PDX1 plays an important role in pancreatic differentiation and insulin gene expression regulation by binding to the A box (A1-A3) and activating the differentiation of pancreas precursor cells into pancreas cells ${ }^{12-16}$. However, this study of ours revealed that PDX1 alone can not induce BMSCs differentiation. Instead, it required the synergy from NKX6.1 for this specific differentiation process, consistent with previous proposal that PDX1 positive precursor cells require certain factors to induce the development of $\beta$ cells. Indeed, PDX1 and NKX6.1 are expressed at the same time during pancreas development ${ }^{9}$ and only the PDX1/NKX6.1 double positive precursor cells can develop into $\beta$ cells. 
Pancreatic $\beta$-cells contain large amount of zinc to form the 2-zinc-insulin hexamer. In this study, the cells in the experimental group showed bright red colour, demonstrating that the induced cells had affluent zinc. The $\beta$-like cell characteristics were further confirmed by the significant expression of insulin that was detected by both RT-PCR.

Many common factors affect the $\beta$-cell development and functions. Glucagon-like peptide-1 (GLP-1) promotes the fetal pancreatic precursor cell differentiation into $\beta$-cells ${ }^{2,3,11,12}$, so does nicotinic amide, working together with $\beta$-cellulin. $\beta$ Cellulin not only promotes the differentiation but also facilitates the formation of pancreatic cell-like aggregates ${ }^{13-15}$. NIC together with B27 promote the differentiation of fetal pancreatic precursor cells and increase the number of $\beta$-cells ${ }^{14-17}$. In addition, $\beta$-mercaptoethanol is a strong oxidant that helps cell aggregation $^{15}$. Therefore, these factors provide a favorable microenvironment for the maturation and function of $\beta$-cells. Indeed, after 7-day treatment with these factors, the insulin secretion level was significantly increased, along with the higher levels of $\mathrm{C}$-peptide than previously reported ${ }^{1-4,16-19}$ and the expression of glucose transporter 2 (GLUT2) ${ }^{12}$, an important molecular marker of $\beta$-cells ${ }^{20,21}$, strongly indicate that these cells were mature and functional. The functionality of these induced cells was confirmed by our animal experiments. In the diabetic mice, the transplantation of these induced cells brought the blood glucose level to normal just three days after the surgery. And the sugar tolerance was also significantly improved.

Single expression of either PDX1, neurogenin 3(NGN3), neurogenic differentiation 1 (NEUROD1), NKX6.1, or hepatocyte nuclear factor (HNF) failed to induce the physiological level of insulin expression in BMSCs, hepatic cells, or entoderm-original cells ${ }^{4,7-10,21-25}$. The co-expression of PDX1 and NKX6.1, however, was highly effective, probably because their synergy activated certain factors such as NGN3 and MafA, which were absent or inactive in the desmohemoblast stem cells. Some negative regulators in the differentiation and development of beta cells may have been inhibited by the co-expression of PDX1 and NKX6.1. However, future investigations are required to identify these factors that are directly involved.

\section{ACKNOWLEDGEMENTS}

This work was supported by the Guangdong provincial Health Department Fund (No. A2011224), the National Natural Science Foundation of China (No. 30973693), the Research Fund for the Doctoral Program of Higher Education of China (No. 20104401110003) and the national high technology research and development program (863 Program) (No. 2011AA02A111).

\section{REFERENCES}

1. R.M. Baertschiger, D. Bosco, P. Morel, V. Serre-Beinier, T. Berney, L.H. Buhler and C. Gonelle-Gispert, Pancreas, 37, 75 (2008).

2. H.W. Wang, L.M. Lin, H.Y. He, F. You, W.Z. Li, T.H. Huang, G.X. Ma and L. Ma, Chin. Med. J., 124, 1534 (2011).

3. E.P. Rafferty, A.R. Wylie, C.T. Elliott, O.P. Chevallier, D.J. Grieve and B.D. Green, Sci. Pharm., 79, 615 (2011).

4. C. Limbert, G. Päth, R. Ebert, V. Rothhammer, M. Kassem, F. Jakob and J. Seufert, Cytotherapy, 13, 802 (2011).

5. D. Gill, K.J. Brocklehurst, H.W. Brown and D.M. Smith, J. Mol. Endocrinol., 47, 59 (2011)

6. Y. Wu, C. Liu, H. Sun, A. Vijayakumar, P.R. Giglou, J. Oppenheimer, R. Qiao, S. Yakar and D. LeRoith, J. Clin. Invest., 121, 2422 (2011).

7. S.Y. Kim and S.G. Rane, Development, 138, 1903 (2011).

8. M. Al-Masri, M. Krishnamurthy, J. Li, G.F. Fellows, H.H. Dong, C.G. Goodyer and R. Wang, Diabetologia, 53, 699 (2010).

9. S. Gefen-Halevi, I.H. Rachmut, K. Molakandov, D. Berneman, E. Mor, I. Meivar-Levy and S. Ferber, Cell Reprogram, 12, 655 (2010).

10. A.C. Binot, I. Manfroid, L. Flasse, M. Winandy, P. Motte, J.A. Martial, B. Peers and M.L. Voz, Dev. Biol., 340, 397 (2010).

11. K.H.H. He, P.I. Lorenzo, T. Brun, C.M. Jimenez Moreno, D. Aeberhard, J.V. Ortega, M. Cornu, F. Thorel, A. Gjinovci, B. Thorens, P.L. Herrera, P. Meda, C.B. Wollheim and B.R. Gauthier, Diabetes, 60, 1705 (2011).

12. K. Chen, X. Yu, K. Murao, H. Imachi, J. Li, T. Muraoka, H. Masugata, G.X. Zhang, R. Kobayashi, T. Ishida and H. Tokumitsu, Metabolism, 60, 579 (2011).

13. W. Donelan, V. Koya, S.W. Li and L.J. Yang, J. Biol. Chem., 285, 12181 (2010).

14. C.W. Hay, L.A. Ferguson and K. Docherty, Biochim. Biophys. Acta, 1769, 79 (2007).

15. X.J. Niu, Z.R. Wang and J. Seufert, Nan Fang Yi Ke Da Хие Хиe Bao, 27, 78 (2007).

16. T. Boonsaen, P. Rojvirat, K.H. Surinya, J.C. Wallace and S. Jitrapakdee, Biochem. J., 405, 359 (2007).

17. A.H. Paz, G.D. Salton, A. Ayala-Lugo, C. Gomes, P. Terraciano, R. Scalco, C.C. Laurino, E.P. Passos, M.R. Schneider, L. Meurer and E. Cirne-Lima, Stem Cells Dev., 20, 223 (2011).

18. A.S. Bernardo, C.H. Cho, S. Mason, H.M. Docherty, R.A. Pedersen, L. Vallier and K. Docherty, Stem Cells, 27, 341 (2009).

19. A. Eshpeter, J. Jiang, M. Au, R.V. Rajotte, K. Lu, J.S. Lebkowski, A.S. Majumdar and G.S. Korbutt, Cell Prolif., 41, 843 (2008).

20. D. Van Hoof, A.D. Mendelsohn, R. Seerke, T.A. Desai and M.S. German, Stem Cell Res., 6, 276 (2011).

21. K.H.H. He, P.I. Lorenzo, T. Brun, C.M. Jimenez Moreno, D. Aeberhard, J.V. Ortega, M. Cornu, F. Thorel, A. Gjinovci, B. Thorens, P.L. Herrera, P. Meda, C.B. Wollheim and B.R. Gauthier, Diabetes, 60, 1705 (2011).

22. E. Hisanaga, K.Y. Park, S. Yamada, H. Hashimoto, T. Takeuchi, M. Mori, M. Seno, K. Umezawa, I. Takei and I. Kojima, Endocr. J., 55, 535 (2008)

23. Z.W. Zhang, L.Q. Zhang, L. Ding, F. Wang, Y.J. Sun, Y. An, Y. Zhao, Y.H. Li and C.B. Teng, FEBS Lett., 585, 2592 (2011).

24. Y.M. Cui, H. Wang, Q.R. Liu, M. Han, Y. Lu and C.Q. Zhao, Bioorg. Med. Chem. Lett., 21, 4400 (2011).

25. R. Saif-Ali, R. Harun, S. Al-Jassabi and W.Z. Wan Ngah, Acta Biochim. Pol., 58, 179 (2011). 